How far, then, should urological investigation proceed in children presenting with a urinary tract infection ? Certainly it seems that urinary infections in boys are more likely to be associated with structural abnormalities of the urinary tract, but that should not lead to a rigid selective policy. Some doctors, indeed, would even claim that there is a strong case for full urological evaluation (including intravenous pyelography and micturating cystography) in any child presenting with a urinary tract infection, regardless of age or sex, but this argument would be as strongly contested by others.

1 Macaulay, D, and Sutton, R N P, Lancet, 1957, 2, 1318.

2 DeLuca, F G, Fisher, J H, and Swenson, O, New England fournal of Medicine, 1963, 268, 75.

${ }^{3}$ Steele, R E, Leadbetter, G W, Crawford, J D, New England fournal of Medicine, 1963, 269, 883.

4 Smallpeice, V, Lancet, 1966, 2, 1019.

${ }^{5}$ Cohen, M, American fournal of Diseases of Children, 1976, 130, 810.

${ }^{6}$ Hallett, R J, Pead, L, and Maskell, R, Lancet, 1976, 2, 1107.

7 Bergström, T, Archives of Disease in Childhood, 1972, 47, 227.

${ }^{8}$ Kass, E H, Transactions of the Association of American Physicians, 1956, 69, 56.

${ }^{9}$ Gower, P E, et al, Archives of Disease in Childhood, 1970, 45, 259.

\section{Diagnosis of allergy}

Hypersensitivity is an abnormal reaction to a foreign substance and is not necessarily immunological in nature. An allergic (atopic) reaction is a form of hypersensitivity that can be shown to be mediated by immune mechanisms. Clinically it is encountered most commonly as allergic rhinitis, asthma, or dermatitis. The allegedly allergic basis for many other diseases makes the precise diagnosis of allergic reactions a matter of clinical importance.

In hypersensitive individuals allergens provoke the formation of specific IgE antibody-a class of immunoglobulin that binds to basophils in the blood and mast cells in the tissues. Combination of the allergen with specific $\operatorname{IgE}$ on the cell surface leads to the release of histamine and other mediators; and, in consequence, skin testing in allergic subjects provokes a weal-and-flare reaction within 15 minutes of challenge. This sequence of events-the immediate hypersensitivity reaction -is the form of immune response that predominates in most allergic conditions. In reality, however, no immune response occurs in isolation, but the different forms are provoked to varying degree by immune stimuli. Each component of the immediate hypersensitivity reaction can be assayed in order to diagnose allergy in general terms or to implicate a particular allergen. In practice, most attention has been given to the estimation of total and antigen-specific IgE in the serum and to provocation tests. ${ }^{1}$

Total serum IgE is measured by the radioimmunoabsorbent technique (RIST), in which antibody to $\operatorname{IgE}$ is bound to Sephadex particles. The concentration of $\mathrm{IgE}$ in the test serum is assayed by its ability to displace a standardised amount of ${ }^{125}$ I-labelled IgE from combining with the antibody. A commercial kit is available for this purpose from Pharmacia. A high concentration of serum IgE points to the probability of an allergic state, making the test clinically useful, ${ }^{2}{ }^{3}$ but the procedure does not indicate which allergen is responsible. In contrast to the other immunoglobulin classes, a high proportion of the total IgE can be accounted for by its reactivity with a single allergen. ${ }^{4-6}$ Unfortunately, the test is not free from technical problems, particularly when measuring low concentrations of IgE. For example, many commercial antisera to IgE react with portions of other immunoglobulins. ${ }^{7}$ Improved methods such as double antibody radioimmunoassay ${ }^{8-10}$ circumvent these difficulties but are not yet in routine clinical use. In the past there have been conflicting reports about the concentration of IgE in the sera of patients with neoplastic and immunodeficiency diseases, but these difficulties were almost entirely methodological in origin. In contrast, reports of raised IgE concentrations in association with cot deaths, ${ }^{11}$ glomerulonephritis, ${ }^{12}$ and connective tissue diseases have been based largely on improved techniques and raise new possibilities about the pathogenesis of these conditions.

The radioallergosorbent test (RAST) is a method of detecting IgE antibody that reacts specifically with suspected allergens. ${ }^{1}$ In principle, the allergen is coupled to an insoluble carrier and is allowed to bind the specific IgE antibody which may be present in the serum of a hypersensitive patient. The bound IgE is measured by its reaction with ${ }^{125}$ I-labelled antibody to IgE. For clinical purposes the assay is usually performed with a commercial kit in which the allergens are coupled to paper discs.

The test has been used clinically to show specific $\operatorname{IgE}$ antibody to a variety of allergens including moulds, pollens, dust, insects, and aspergilli. ${ }^{13}$ More recently it has been applied to the diagnosis of allergy to drugs such as rifampicin ${ }^{14}$ and to food antigens such as milk, ${ }^{15}$ eggs, and wheat. ${ }^{16}$ In patients with atopic dermatitis it has been valuable in indicating the responsible allergen. ${ }^{16}$ The correlation between the RAST test on the one hand and the clinical features and skin tests on the other varies in different disorders. With pollens it is good, ${ }^{6} 1718$ but for animal danders and house dust it is much less satisfactory. ${ }^{19}$ Moreover, false-positives are commonly encountered in RAST tests for food allergens, ${ }^{19}{ }^{20}$ and the results may be inappropriate to the extent of the clinical problem-for example, RAST tests may not distinguish patients with atopic dermatitis and allergic rhinitis who develop asthma from those who do not. ${ }^{21} 22$

The standard RAST test suffers from several disadvantages: variable binding properties of IgE antibody in different sera, interference by specific antibody of classes other than $\operatorname{IgE}, 2324$ and the non-standardised units in which the results must necessarily be expressed. ${ }^{5}$ Improved methods include measuring IgE antibody alone, bound to the allergen free from antibodies of other classes, ${ }^{5}$ and the introduction of highly purified antibody to IgE in the final stages of the assay. ${ }^{25}$ Soon enzymelinked reagents are likely to replace radiolabelled materials. Such refinements improve both the specificity and the sensitivity of the RAST technique; but the method, although expensive, is basically safe, easy to perform, and little affected by concurrent treatment.

In contrast, conventional skin tests can be performed simply, cheaply, and without delay but carry the risk of provoking anaphylaxis. ${ }^{13} 26$ Highly purified allergen preparations induce responses that are both sensitive and specific, ${ }^{27}$ but skin testing with reagents in general use produces less useful results. Positive reactions may have little significance: in one survey over $40 \%$ of normal individuals in certain age groups produced reactions to allergens in dusts and moulds, ${ }^{28}$ and clearly most of these were clinically unimportant. Conversely, skin tests may be unexpectedly negative.

Local secretion of specific IgE may predispose to allergic disease in patients in whom the responsible allergen does not elicit positive skin reactions. ${ }^{29}$ Certainly provocation tests may induce a reaction only when the appropriate tissue is challenged -for example, allergic responses in the respiratory tract are 
best provoked by inhalation, ${ }^{30}$ while dietary manipulation is currently the most effective way of evincing food allergy. ${ }^{31}$ The solution to many of these problems lies in the introduction of pure, carefully characterised allergens for both skin testing and assays of specific IgE antibody. ${ }^{32} 33$ "Allergy" has been a subject of confusion in the past and will not be clarified even now without rigorous standardisation. A good example of the perils of allergic controversy was the recent suggestion that milk allergy contributes to myocardial infarction; this was firmly refuted by prompt recourse to the RAST test. ${ }^{34}$

${ }^{1}$ Foucard, T, in Progress in Immunology, Vol 4, ed L Brent and E J Holborow, p 320. Amsterdam, North Holland, 1974.

2 Berrens, L, and Bruynzeel, P, Clinica Chemica Acta, 1970, 70, 337.

3 Turner, K J, Rogman, D L, and O'Mahoney, J, International Archives of Allergy, 1974, 47, 650 .

${ }^{4}$ Gleich, G J, and Jacob, G L, Science, 1975, 190, 1106.

${ }^{5}$ Schellenberg, R R, and Adkinson, N F, fournal of Immunology, 1975, 115, 1577.

${ }^{6}$ Church, J A, Kleban, D G, and Bellarti, A, Pediatric Research, 1976, 10, 97.

7 Bernier, G M, and McIntyre, O R, fournal of Immunological Methods, 1976, 13, 91.

${ }^{8}$ Gleich, G M, et al, fournal of Laboratory and Clinical Medicine, 1971, 77, 690.

${ }^{9}$ Polmar, S H, Waldmann, T A, and Terry, D, fournal of Immunology, $1973,110,1253$.

${ }^{10}$ Buckley, R H, and Fiscus, S A, fournal of Clinical Investigation, 1975, 55, 157.

11 Turner, K J, Baldo, B A, and Hilton, J M N, British Medical fournal, 1975, $1,357$.

12 Faleroni A, E Earle, D P, and Patterson, R, fournal of Chronic Diseases, 1976, 29, 599.

13 Yunginger, J W, and Gleich, G J, Pediatric Clinics of North America, 1975, 22, 3 .

14 Bassi, L, Di Berardino, L, and Silvestri, L G, International Archives of Allergy and Applied Immunology, 1976, 51, 390.

15 Hoffman, D R, and Haddad, Z H, Fournal of Allergy and Clinical Immunology, 1974, 54, 165 .

16 Caldwell, J H, Tennenbaum, J I, and Bronstein, H A, New England Fournal of Medicine, 1975, 292, 1388.

17 Berg, T, Bannich, H, and Johansson, S G, International Archives of Allergy, 1971, 40, 770.

18 Lichtenstein, L M, et al, fournal of Clinical Investigation. 1973, 52, 472.

19 Mattikow, M S, Lancet, 1976, 1, 1414.

${ }^{20}$ Foucard, T, Acta Paediatrica Scandinavica, 1973, 62, 633.

21 Jones, H E, et al, British fournal of Dermatology, 1975, 92, 17.

22 Bruce, C A, et al, Clinical Experimental Immunology, 1976, 25, 67.

23 Bongrand, P, et al, fournal of Immunological Methods, 1976, 11, 197

${ }^{24}$ Lynch, N R, et al, Clinical Experimental Immunology, 1975, 22, 35.

25 Goodwin, B F J, and How, M J, Clinical Allergy, 1976, 6, 441.

${ }^{26}$ Baldo, B A, and Turner, K J, Medical fournal of Australia, 1975, 2, 871.

27 Aas, K, and Johansson, S G O, Fournal of Allergy and Clinical Immunology, $1971,48,134$.

${ }^{28}$ Barbee, R A, et al, Annals of Internal Medicine, 1976, 84, 129

${ }^{29}$ Huggins, K J, and Brostoff, J, Lancet, 1975, 2, 148.

30 Pepys, J, New England fournal of Medicine, 1975, 293, 758

31 Chua, Y Y, et al, fournal of Allergy and Clinical Immunology, 1976, 58, 299.

32 Aas, K, International Archives of Allergy and Applied Immunology, 1975, 49, 44 .

33 World Health Organisation, fournal of Biological Standards, 1975, 3, 113.

34 Toivanen, A, Viljaren, M K, and Savilahti, E, Lancet, 1975, 2, 205.

\section{Potassium in heart failure}

Some $98 \%$ of the total $3500 \mathrm{mmol}$ of body potassium is located in the intracellular compartment, where it forms the major cation. Only $2 \%$ is found in extracellular fluid, but this small amount is important: its ratio to intracellular potassium determines the transmembrane potential on which depends the normal functioning of cells, especially nerve and muscle. Hypokalaemia can cause myocardial dysfunction, neuromuscular weakness, psychiatric syndromes, and proximal renal tubular damage with a reduction in concentrating ability, ${ }^{1}$ and it predisposes to digitalis intoxication. ${ }^{2}$ Potassium balance is, therefore, of vital importance to patients with heart disease and has been studied widely.

Studies of patients in heart failure have shown that most are depleted of total exchangeable potassium, despite normal plasma concentrations..$^{3-5}$ The explanations include anorexia, poor diet, and increased urinary loss due to hyperaldosteronism. Furthermore, many patients have a reduced body cell mass, and some who are hypoxic or acidotic develop a "sick cell syndrome," in which intracellular sodium concentrations rise as potassium falls. In these circumstances supplements do not restore exchangeable cation, and the condition is better termed potassium depletion. ${ }^{6}$ Diuretics, both of the benzothiadiazine series and of the potent loop type (such as frusemide, ethacrynic acid, and bumetanide), increase the sodium content of filtrate reaching the distal convoluted tubule and produce a hypochloraemic alkalosis because of exchange between sodium ions and hydrogen or potassium in this region. They also predispose to digitalis intoxication. ${ }^{7}$

These factors make potassium supplements (or prevention of urinary potassium loss) mandatory in patients who are receiving both digitalis and diuretics, in elderly patients on diuretics alone (because their intake is poor $^{8}$ ), and in those who require cardiac surgery. There is, however, little evidence to support their indiscriminate use, ${ }^{9}$ and recent studies ${ }^{10}$ have suggested that they may sometimes be more hazardous than beneficial. Supplements should ideally be given in the diet, but commercial fruit drinks often do not contain adequate amounts of potassium and fresh fruit and vegetables are beyond the means of many elderly patients.

Additional potassium can be supplied in tablets. The early preparations produced gastric irritation; this was circumvented by the introduction of an enteric-coated form, but rapid dissolution of such tablets led to high concentrations of potassium in the intestine, causing ulceration of the bowel with subsequent perforation or stenosis. ${ }^{11}$ Enteric-coated potassium preparations, including those combined with a diuretic, should no longer be used. Slow-K is a more suitable tablet preparation: the potassium is encased in a wax matrix and is released relatively slowly. Some patients find these tablets difficult to swallow, however, and they should not be given to patients with advanced mitral valve disease and enlargement of the left atrium, as oesophageal stenosis may result. ${ }^{12}$ Intestinal stenosis due to Slow-K is, however, rare unless transit time is prolonged ${ }^{13}$ or the tablet is held up by an anatomical abnormality. Potassium can also be supplied in effervescent forms. The original preparations did not contain chloride, and as a result failed to correct the associated metabolic alkalosis, ${ }^{14} 15$ but effervescent formulations containing chloride have now been developed: these include Kloref and Sando-K. In addition to correcting the metabolic alkalosis these are usually more effective in restoring potassium balance than preparations that lack chloride.

Opinions differ on the amount of supplementary potassium required. Edmonds ${ }^{16}$ has suggested that a dose of $24 \mathrm{mmol} /$ day is sufficient for most hypertensive patients, whereas White ${ }^{17}$ used up to twice this amount to raise the exchangeable potassium in patients with heart failure. In patients who cannot tolerate potassium supplements and those whose oedema is resistant to high doses of frusemide it is sensible to use a potassium-conserving diuretic as well as one that acts on the loop of Henle. Of the three drugs available, spironolactone is a competitive aldosterone antagonist, whereas amiloride and triamterene are thought to occupy sodium channels in the distal convoluted tubule, thereby preventing its reabsorption. All three potentiate sodium excretion and 